\title{
Comparación de criterios para determinar la edad de adquisición de fonemas lateral y róticos
}

\section{Comparison of Criteria for Determining the age of Acquisition of Lateral Phonemes and Rhotic}

\section{Felipe Torres M. \\ Fonoaudiólogo \\ Universidad de Chile}

\section{Josué Pino $\mathrm{C}$.}

Lic. en Letras

Universidad de Chile

\section{Christian Peñaloza C.}

Lic. en Letras

Universidad de Chile

\section{Daniela Rojas C. \\ Fonoaudióloga \\ Universidad de Chile}

\section{María Angélica Fernández G. \\ Fonoaudióloga \\ Universidad de Chile \\ Patricia Castro A. \\ Fonoaudióloga \\ Universidad de Chile \\ Mario Bustos R. \\ Fonoaudiólogo \\ Universidad de Chile}

\section{Katerine Hormazábal M.}

Fonoaudióloga Santiago, Chile

\begin{abstract}
RESUMEN
El desarrollo fonético-fonológico es abordado de diferentes formas cuando se estudia el tema. Una de ellas se basa en el establecimiento de edades de adquisición de los fonemas de una lengua. Una revisión de la literatura al respecto evidencia que las distintas investigaciones en esta temática no siempre utilizan los mismos criterios en el análisis de los datos, en la selección de la muestra o en el juicio clínico para definir adquisición, entre otros puntos relevantes. Por esta razón, los objetivos del presente artículo son comparar distintos criterios de análisis y determinar de qué manera la elección de uno u otro influye en la edad de adquisición de los fonemas estudiados. Para llevar a cabo los objetivos, se evaluaron 84 niños de entre 3 años y 4 años 11 meses, organizados en cuatro grupos de 21 niños según rango etario. En todos ellos se evaluó la producción de los fonemas lateral / I / y róticos / r / y / r / en distintos contextos fonológicos. Luego, a la muestra de fonemas obtenida, se le aplicaron los diferentes criterios que se pretendía comparar. Los resultados muestran que la edad de adquisición de los fonemas estudiados varía según el criterio utilizado, incluso en algunos casos existen al menos dos años de diferencia. A partir de los resultados, se discute la importancia de explicitar los criterios que se utilizan para estudiar edades de adquisición y, sobre todo, la implicancia que puede tener seleccionar uno u otro criterio en el ejercicio fonoaudiológico.
\end{abstract}

Palabras clave: contextos fonológicos, criterios de adquisición, desarrollo fonológico, edad de adquisición, emergencia del fonema, estabilización del fonema.

\begin{abstract}
Phonological development is approached in different ways in the literature. One is based on the establishment of the age of acquisition of the phonemes of a language. A review of the literature showed that in this area researchers do not always use the same criteria in the analysis of data in the selection of the sample or clinical judgment to determine acquisition, among other important points. Therefore, the aim of this study is to compare different approaches and determine how the choice of one or the other influences the age of acquisition of the phonemes studied. To accomplish the objectives, 84 children aged between 3 and 4 organized into 4 groups of 21 children were evaluated according to age range. They were evaluated in the production of the lateral / I / and rhotic phonemes / r / and / $r$ / in different phonological contexts. Then different criteria were applied to the sample of obtained phonemes to compare them. The results showed that the age of acquisition of phonemes studied varies according to the criteria used, in some cases up to two years apart. From the results, there is a discussion of the importance of explaining the criteria used when considering the age of acquisition and especially the implications it may have when different criteria is chosen for a speech therapy exercise.
\end{abstract}

Keywords: acquisition criteria, age of acquisition, phoneme emergence, phoneme stabilization, phonological context, phonological development.

\section{Contacto con el autor: Felipe Torres M. Santiago - Chile Correo-e: felipefonoaudiologo@gmail.com}




\section{Introducción}

Una etapa importante en la evaluación fonoaudiológica es la valoración fonética-fonológica del desempeño del niño. Al realizarla, los especialistas requieren información acerca de las edades de referencia en las que se adquieren los fonemas del español en el desarrollo típico, tanto para establecer un diagnóstico como para dar una descripción específica que luego oriente el proceso terapéutico. En español se cuenta con una serie de propuestas de edades de referencia, desde los trabajos pioneros de Bosch (1983), Melgar (1976/2007) y Serra (1984), hasta los últimos aportes de Susanibar, Dioses y Huamaní (2013). Dado que aún existen pocos trabajos que informen respecto de las edades de referencia para el español de Chile, se consideran todas las aportaciones hispanoamericanas como un solo conjunto de referencia, aunque esto siempre deba tomarse con precaución, dadas las diferencias en los repertorios fonológicos entre el español americano y el peninsular.

Los estudios disponibles suelen coincidir en algunos resultados, como por ejemplo en un orden general de adquisición, con los fonemas nasales y oclusivos áfonos como los más tempranos, hasta algunos fricativos y los róticos como los más tardíos. Sin embargo, las edades más específicas que se proponen para cada fonema en las distintas investigaciones pueden coincidir o no, producto de consideraciones y decisiones teóricas y metodológicas que no siempre son explicitadas y/o discutidas en los trabajos. Estos aspectos son importantes de clarificar, dado el uso clínico que se realiza de la información propuesta y, en general, para entender cómo se obtuvieron los datos y qué perspectivas manejaron los distintos autores en relación con la adquisición de los fonemas. Entre las diferencias presentes se pueden mencionar asuntos metodológicos (como el número de veces que se le solicitó a cada niño emitir un mismo sonido) y teóricos (como si se establece o no una distinción entre lo fonético y lo fonológico). Igualmente, resultan relevantes algunos aspectos que suelen no discutirse, como las implicancias de los criterios utilizados para conformar la muestra o en la adopción de un diseño transversal de investigación, utilizado por todos los trabajos consultados.

Considerando estas diferencias, el presente estudio se propone profundizar en tres aspectos que resultan especialmente importantes. El primero de ellos se relaciona con la manera en la que se procesa el conjunto de datos obtenidos; el segundo, con el uso (o no) de un criterio para indicar que un sonido se encuentra adquirido; y el tercero, con la diferenciación (o no) que se efectúa de las posiciones que el fonema puede ocupar dentro de la sílaba y/o palabra. Las decisiones que se tomen en cada uno de estos aspectos van definiendo cómo se ha conceptualizado el objeto de estudio y la posibilidad de replicar y proyectar los resultados, por ejemplo, en la actividad clínica. A continuación, se revisa cada uno de estos puntos y se expone qué decisiones, explícitas o implícitas, han tomado las investigaciones disponibles acerca de la adquisición de los fonemas en español. 


\section{a) Procesamiento de los datos}

El primer aspecto está relacionado con la manera de procesar los datos obtenidos, frente a lo cual se observan dos modalidades: un análisis grupal de todas las emisiones por fonema, o bien, un análisis niño por niño. En el primero de los casos, se informa el porcentaje de emisiones correctas o erróneas, según el modelo adulto, de cada unidad por cada grupo de edad contemplado en la investigación. De esta manera procede Acevedo (1993) para el español de niños mexicanos migrantes en Estados Unidos; Melgar (1976/2007), para el español de México; Serra (1984), para el español peninsular; y Vivar (2009) y Vivar y León (2009), para el español de Chile. Esta modalidad busca, principalmente, aminorar el impacto de las diferencias individuales en los resultados globales (Vivar y León, 2009) y el foco de análisis se centra en "el comportamiento del sistema lingüístico [más que en] la población" (Vivar, 2009, p. 44); es decir, se asume que cada fonema presenta características propias que facilitan o no su adquisición a cierta edad, en particular su conjunto de rasgos. En este artículo, se hará referencia a estos trabajos como investigaciones centradas en el fonema.

En el segundo de los casos, las investigaciones informan el porcentaje de niños que tienen adquirido (o no) cada unidad por cada grupo de edad. De esta manera procede Bermeosolo (2001), Hormazábal, Larrea, Muñoz, Ruz y Toro (2013) y Jara, Poblete, Riquelme, Salgado y Zúñiga (2014), para el español de Chile; Bosch (1983), para el español peninsular; Camargo (2006), para el español de Colombia y Susanibar et al. (2013) para el español de Perú. En estos casos, el foco está puesto en la cantidad de sujetos de una misma edad que manifiestan adquirida cada unidad, lo que presupone que son ellos, y ya no los fonemas en sí, los que presentan características por ejemplo de maduración, que podrían incidir en su nivel de dominio fonológico (Bosch, 1983). En el presente artículo se hará referencia a este tipo de trabajo como investigaciones centradas en los sujetos.

\section{b) Criterios de adquisición}

La mayoría de las investigaciones propone distinguir, a través de un porcentaje, entre adquirido y no adquirido. Este porcentaje puede ir desde el $65 \%$ al $100 \%$, ya sea del total de veces que un fonema es correctamente emitido por un grupo, o bien, del total de niños que ya lo tendría en su repertorio. Lo anterior deja de manifiesto que, aunque los trabajos marquen como hito un mismo porcentaje, los resultados pueden ser no equiparables.

En concreto, las investigaciones centradas en el fonema distinguen entre un sonido adquirido de uno no adquirido, de acuerdo con el porcentaje de emisiones correctas en relación con el total del grupo. Melgar (1976/2007) utiliza como hito el 90\% de emisiones correctas por grupo; Vivar y León (2009) resaltan aquellos que alcanzan un $100 \%$ de emisiones correctas ya a los 3 años. Acevedo (1993), por su parte, califica un sonido como adquirido cuando alcanza el $90 \%$ de emisiones correctas, siempre que dicho porcentaje se observe en dos grupos de edad consecutivos. En tanto que Vivar (2009), al analizar la emisión de grupos consonánticos, sugiere que el $80 \%$ de emisiones correctas diferencia a los grupos adquiridos de los 
que aún no los son. Un problema con estos trabajos es que no explicitan qué criterios fueron considerados para optar por un porcentaje en vez de otro.

En las investigaciones centradas en los individuos, no siempre se opta por definir un punto de referencia para considerar adquirido un fonema. Bermeosolo (2001), Bosch (1983), Camargo (2006), Hormazábal et al. (2013), Jara et al. (2014) y Susanibar et al. (2013), informaron del porcentaje de niños que manifiesta adquirido o no por grupo. Sin embargo solo Camargo (2006), Jara et al. (2014) y Susanibar et al. (2013) precisan un porcentaje específico (Camargo, un 65\%; Jara el al. y Susanibar et al. un 75\%), Tampoco se justifica por qué se opta por estas cifras. Sin embargo, en estas investigaciones el asunto crucial es otro: qué criterio se utilizó para definir que un niño tiene adquirido o no un determinado fonema. Bermeosolo (2001), Bosch (1983) y Camargo (2006) no lo explicitan, aunque se entiende que el fonema se consideró adquirido cuando el niño lo emitió en todas las ocasiones que se le solicitó; Hormazábal et al. (2013) calificaron que un niño tenía adquirido un sonido cuando lo emitía en, al menos, un $80 \%$ de las veces que se le pedía; en cambio, Jara et al. (2014) optaron por el 75\%; por su parte, Susanibar et al. (2013) explicitan que el sonido se encuentra adquirido cuando es articulado correctamente en las distintas posiciones de la palabra. Nuevamente, los autores no justifican la razón de escoger el porcentaje respectivo.

La importancia de clarificar el criterio para seleccionar un porcentaje $u$ otro, o ninguno en algunas investigaciones, radica en que dicha decisión podría dar cuenta de la concepción que los autores tienen de la noción de adquisición, es decir, qué alcance y estabilidad deben presentar los datos para que un fonema sea definido como adquirido. Además, el uso de un porcentaje, independientemente de cuál sea este, da cuenta de que las investigaciones en español tienden a operacionalizar el fenómeno de manera dicotómica (adquirido/no adquirido), y no abordarlo de un modo progresivo, en que se diferencie, por ejemplo, entre la emergencia y el dominio de un fonema; esto, a pesar de que los autores insisten en que sus resultados no representan etapas de adquisición rígidas (Bosch, 1983; Melgar 1976/2007; Vivar y León, 2009). Quizás el único fenómeno descrito que permite dar cuenta, parcialmente, de cierta inestabilidad en el proceso de adquisición es de la inversión (reversal), mencionado en algunos estudios centrados en el fonema. Este fenómeno ocurre en los casos en que una unidad alcanza, en un grupo de edad, el porcentaje que permite considerarlo como adquirido y no logra alcanzarlo en el grupo de edad siguiente (Acevedo, 1993); es decir, cuando no se observa el aumento progresivo lineal esperado de antemano. Vivar y León (2009) informan de este comportamiento en algunos fonemas, específicamente en los líquidos, al comparar el número de emisiones correctas en grupos de edades consecutivas. En tanto Vivar (2009) observa el mismo fenómeno al considerar la emisión de grupos consonánticos.

\section{c) Posiciones del fonema dentro de la palabra}

También resulta crucial en este tipo de investigaciones conocer si al obtener los datos se 
consideraron distintas posiciones del fonema dentro de la palabra y si los resultados están reportados para cada contexto fonológico (fonema en posición de ataque, grupo consonántico u otro). Todas las investigaciones consultadas prepararon listados de palabras para elicitar los fonemas en los sujetos, excepto Camargo (2006), quien utilizó lenguaje espontáneo. En el caso de los listados, todos los investigadores trabajaron con palabras que presentaran el fonema en posición de onset en inicio de palabra y en posición medial. En el caso de fonemas en posición de coda, algunos explicitan que solo la midieron al final de la palabra (Acevedo, 1993; Melgar, 1976/2007); otros, en medio y al final de la palabra (Bermeosolo, 2001; Hormazábal et al., 2013; Jara et al., 2014; Susanibar et al., 2013), y otros no precisan información al respecto (Bosch, 1983; Serra, 1984). Por último, todos los trabajos incluyen palabras con grupos consonánticos, excepto Acevedo (1993). En resumen, la mayoría de las investigaciones contemplaron la aparición del fonema en tres o más posiciones dentro de la palabra. En la entrega de los resultados, sin embargo, no siempre se diferenciaron los contextos utilizados: Melgar (1976/2007), Serra (1984) y Camargo (2006) entregaron resultados generales por fonema; Bosch (1983), Bermeosolo (2001), Vivar (2009) y Vivar y León (2009) diferencian sus resultados para onset más coda, por una parte, y grupos consonánticos, por otra. Por su parte, Hormazábal et al. (2013) y Jara et al. (2014) distinguieron entre ataque silábico y coda, mientras que Acevedo (1993), diferencia, además, entre ataque silábico al inicio de la palabra o al interior de ella. Finalmente, Susanibar et al. (2013) distinguen entre inicio silábico al principio de la palabra, así como al interior de la misma, tanto para los sonidos en ataque silábico, como en grupos consonánticos. Esta variabilidad exige poner mucha atención al momento de interpretar los resultados. Por ejemplo, si se consideran las investigaciones con foco en el fonema, Acevedo (1993) establece que el fonema / I / es emitido correctamente el $100 \%$ de las veces por el grupo de 3 años en posición de ataque inicial; mientras que Vivar y León (2009), lo establecen a los 4 años 6 meses, pero considerando posición de ataque y coda a la vez.

En resumen, los tres aspectos descritos anteriormente permiten visualizar que las distintas investigaciones ofrecen diversos criterios para procesar el dato fonético-fonológico obtenido a partir de sus muestras $y$, por lo tanto, hacen referencia a fenómenos distintos cuando entregan sus resultados, aunque estos parezcan similares. En esa línea, los objetivos de este trabajo son, por un parte, comparar las distintas opciones que se seleccionan para procesar los datos, en la toma de un criterio de adquisición y en la diferenciación de las posiciones del fonema dentro de la palabra; y por otra, se pretende evidenciar el impacto que genera sobre la información de referencia que se ofrece de cada fonema. Esto es con el propósito de discutir las implicancias que puede tener el seleccionar uno $u$ otro criterio en la aplicación que hacen de estos datos los fonoaudiólogos chilenos. En concreto, se propone procesar los datos de una misma muestra de producción fonético-fonológica del español de Chile, en este caso del fonema lateral / I / y de los fonemas róticos: vibrante simple / $\mathrm{r} / \mathrm{y}$ vibrante múltiple / $\mathrm{r} /$, con algunos de los distintos criterios utilizados en los trabajos referenciados y, a partir de 
esos distintos análisis, valorar el tipo de resultados que se obtienen.

\section{Metodología}

Se efectúa un estudio descriptivo, con diseño no experimental y transversal en el que se consideró como variable independiente la edad de los participantes y como variable dependiente, la producción de los fonemas róticos y lateral en diferentes posiciones de una cadena fónica ${ }^{1}$.

\section{a) Participantes y criterios de selección}

Los criterios de inclusión fueron: ser chileno, monolingües del español de Chile, vivir en la Región Metropolitana Santiago de Chile, tener entre 3.00 a 4.11 años y pertenecer al nivel socioeconómico medio. Este último se definió según la distribución socioeconómica de la Región Metropolitana del Instituto Consultor en Comercialización y Mercado (ICCOM), más el cumplimiento de dos de los siguientes tres criterios propuestos por el mismo instituto: ocupación, profesión y educación del “jefe de hogar". La información se consultó a los padres a través de un cuestionario.

La muestra quedó compuesta por 84 niños chilenos de entre 3.00 a 4.11 años (39 niñas y 45 niños) asistentes a jardines infantiles de las comunas de Maipú y La Florida. Para los análisis, la muestra se dividió en cuatro grupos de 21 niños, según rangos

\footnotetext{
1 Se realiza un reanálisis de los resultados del seminario de investigación "Edad de adquisición de fonemas líquidos en un grupo de niños chilenos entre 3 y 4 años 11 meses de nivel socioeconómico medio en la Región Metropolitana" (Hormazábal et al., 2013) para obtener el grado de Licenciado en Fonoaudiología, Universidad de Chile, tutoreado por María Angélica Fernández. Dicho seminario se realizó en el marco del Proyecto 145, Departamento de Fonoaudiología, Universidad de Chile dirigido por Christian Peñaloza, como investigador responsable.
}

de edad de 6 meses cada uno: 3.00 - 3.05, 3.06 $3.11,4.00-4.05$ y $4.06-4.11$. En todos los casos, los padres firmaron un consentimiento informado validado por el Comité de Ética de la Facultad de Medicina de la Universidad de Chile.

\section{b) Procedimientos de evaluación}

Las muestras fonético-fonológicas obtenidas se recopilaron sobre la base de dos pruebas de producción. La primera, consistió en la elicitación de sílabas aisladas que contuvieran los fonemas estudiados en posición inicial prevocálica ("la”) y en posición inicial intervocálica ("ala"), las cuales los niños emitían después de ser dichas por la evaluadora. El fonema / r / solo se evaluó en posición intervocálica. En la segunda prueba, se trabajó con una serie de 39 láminas con imágenes representativas de palabras que contenían los fonemas estudiados en diferentes posiciones de la cadena fónica: ataque silábico, coda y grupo consonántico. La instrucción que entregó la evaluadora fue: "Ahora vamos a ver unas imágenes y tú me tienes que decir qué son. Mira esta imagen, ¿qué es?". Si el niño no decía la palabra esperada o no enunciaba sonido alguno, se le daban pistas semánticas de la imagen. Si aún no se obtenía la palabra, entonces se procuraba realizar una repetición diferida. Finalmente, si esto no daba resultado, se realizaba una repetición directa.

En total, cada niño emitía un máximo de 31 / I / (diez en sílabas aisladas, cinco prevocálicas y cinco intervocálicas, ocho en posición de ataque en palabra, dos en posición de coda y once en grupo consonántico), 28 / r / (cinco en sílaba intervocálica aisladas, cinco en posición de ataque en palabra, 
cuatro en posición de coda y 14 en grupo consonántico) y 12 / r / (diez en sílabas aisladas, cinco prevocálicas y cinco intervocálicas y dos en posición de ataque en la palabra).

Las respuestas de cada niño fueron grabadas en audio y registradas en los protocolos de cada prueba. En caso de duda, la respuesta del niño fue analizada al menos por dos evaluadores.

Cabe explicitar que se utilizaron dos pruebas para obtener los datos, una de sílabas aisladas y otra de palabras, ya que la producción de estas últimas puede verse interferida por la presencia de procesos fonológicos de simplificación que presenten los niños de la muestra (Susanibar et al., 2013). Ambas pruebas fueron creadas para efectos de esta investigación y se seleccionaron los estímulos considerando la familiaridad léxica y la factibilidad de ser representados por una imagen. Los estímulos gráficos fueron realizados por un dibujante, en formato blanco y negro, y donde solo se representó la palabra en cuestión. Antes de la aplicación de las pruebas se realizó un pilotaje a 16 niños de un jardín infantil, ubicado en la comuna de Recoleta, con la obtención del consentimiento informado por parte de los padres. Este proceso mostró que el material era pertinente tanto en su implementación, como también en el contenido del mismo.

\section{c) Procesamiento de los datos}

En este trabajo los datos se procesaron utilizando dos criterios que concuerdan con la literatura: un análisis centrado en el fonema y un análisis centrado en los sujetos.

Por una parte, en el análisis centrado en el fonema, se calculó el total de veces que fueron producidos de manera efectiva los fonemas / I /, / r / y / r / considerando la posición dentro de la palabra. En tanto que en el análisis centrado en los sujetos, se calculó el total de niños que produjo el fonema. Para establecer que el niño producía el segmento, se utilizaron dos criterios individuales: en primera instancia, el criterio de evidencia de producción, esto es "dice el fonema al menos una vez, incluido siempre", utilizado por Dodd et al. (2003) en una investigación acerca del inglés británico; y en segundo término, la distinción entre inestabilidad y estabilidad, esto es "dice el fonema al menos una vez, pero no siempre" y "dice el fonema siempre", respectivamente.

Finalmente, en ambos tipos de análisis (el centrado en el fonema $\mathrm{y}$ aquel centrado en los individuos), se aplicaron dos criterios de adquisición (80\% y 90\%). Estos valores porcentuales se escogieron de forma arbitraria y solo para ejemplificar cómo varía la edad de adquisición que se establece, al considerar uno u otro porcentaje.

\section{Resultados}

Los resultados se presentan considerando los distintos criterios que se pretenden comparar. En primera instancia, los datos se muestran separados según criterio de análisis, esto es, centrado en el fonema y centrado en los individuos. En cada uno de ellos, se comparan los resultados al utilizar diferentes porcentajes de adquisición y en distintos contextos fonológicos. Finalmente, se contrastan las edades de producción al emplear uno u otro criterio de adquisición. 


\section{a) Resultados por fonema}

Los datos que se presentan a continuación evidencian la cantidad de fonemas producidos en cada rango etario, expresados en porcentajes. La Tabla 1 ilustra el panorama general para los tres fonemas estudiados y luego las Tablas 2,3 y 4 muestran los resultados para cada fonema, en relación con los diferentes contextos fonológicos (en sílabas aisladas y dentro de palabras en posición de ataque, de coda y en grupo consonántico).

Tabla 1

Porcentajes de producción de fonemas por rango etario sin distinción de contextos fonológicos (entre paréntesis se señala el número de emisiones posibles de cada fonema)

\begin{tabular}{cccc}
\hline Rango etario & $\mathrm{l}$ & $\mathrm{r}$ & $r$ \\
& $(100 \%=651)$ & $(100 \%=588)$ & $(100 \%=252)$ \\
\hline $3.00-3.05$ & $60,4 \%$ & $31,6 \%$ & $16,7 \%$ \\
$3.06-3.11$ & $72,2 \%$ & $42,5 \%$ & $29,0 \%$ \\
$4.00-4.05$ & $90,0 \%$ & $75,7 \%$ & $68,7 \%$ \\
$4.06-4.11$ & $92,6 \%$ & $87,6 \%$ & $64,3 \%$ \\
\hline
\end{tabular}

Como se advierte en la Tabla 1, a mayor edad, mayor es el porcentaje de aparición de los fonemas, con excepción del fonema vibrante múltiple / r / para el rango etario de los $4.06-4.11$, donde se observa una disminución en el porcentaje de producción. Por otra parte, en todos los rangos etarios evaluados el fonema lateral / I / es producido una mayor cantidad de veces respecto de los fonemas róticos $y$, entre estos, el fonema vibrante simple / $\mathrm{r} /$ es emitido en mayor cantidad respecto del vibrante múltiple / $\mathrm{r} /$, lo que coincide con la secuencia de aparición que se describe en la literatura.

Al aplicar a dichos resultados un criterio de adquisición que tome como punto de corte el $80 \%$ de emisiones correctas por grupo, se obtiene que el fonema / I / se adquiere a los 4 años de edad; / r / a partir de los 4 años 6 meses; y / r / después de los 5 años. En cambio, si se aplica el criterio del 90\%, / I / no cambiaría su edad de adquisición, pero sí los fonemas róticos, pues ninguno alcanza un $90 \%$ de emisiones correctas antes de los 4 años 11 meses.

A continuación, se ofrecen los resultados para cada fonema, según las distintas posiciones en que aparecen dentro de las sílabas aisladas (prevocálicas e intervocálicas) y las palabras (ataque, coda y grupo consonántico).

Tabla 2

Porcentajes de producción del fonema / / por rango etario con distinción de contextos fonológicos (entre paréntesis se señala el número máximo de producciones en cada contexto)

\begin{tabular}{ccccc}
\hline & \multicolumn{4}{c}{ Fonema / I/ } \\
\cline { 2 - 5 } Rango etario & Sílabas aisladas & \multicolumn{3}{c}{ Palabras } \\
\cline { 2 - 5 } & $(100 \%=210)$ & Ataque & Coda & Grupo consonántico \\
& & $(100 \%=168)$ & $27,7 \%$ & $66,7 \%$ \\
& $86,7 \%$ & $70,8 \%$ & $53,7 \%$ & $76,2 \%$ \\
$3.00-3.05$ & $86,2 \%$ & $79,2 \%$ & $81,8 \%$ & $92,9 \%$ \\
$4.00-4.05$ & $97,1 \%$ & $91,7 \%$ & $82,3 \%$ & $90,5 \%$ \\
$4.06-4.11$ & $100,0 \%$ & $98,2 \%$ & & \\
\hline
\end{tabular}


Tal como se observa en la Tabla 2, la producción del fonema lateral / I / muestra porcentajes de aparición más altos en sílabas aisladas que en los contextos fonológicos de palabra en los distintos rangos etarios. La secuencia de mayor a menor porcentaje de producción según contexto fonológico es: sílabas aisladas, palabras en posición ataque, en grupo consonántico y, por último, en posición de coda. Además, en cada contexto se observan porcentajes ascendentes de emisión correcta a medida que aumenta la edad de los participantes. Al aplicar el criterio de adquisición del $80 \%$, se obtiene que el fonema / I / se encontraría adquirido en cualquier contexto a partir de los 4 años de edad, incluso a partir de los 3 en el contexto de sílaba aislada. En cambio, al aplicar el criterio del 90\%, todos los contextos alcanzan dicho porcentaje en el grupo de los 4 años, excepto en posición de coda, que no alcanza el punto de corte en ninguna edad de la muestra.

Tabla 3

Porcentajes de producción del fonema / $/$ / por rango etario con distinción de contextos fonológicos (entre paréntesis se señala el número máximo de producciones en cada contexto)

\begin{tabular}{ccccc}
\hline & \multicolumn{4}{c}{ Fonema / $\mathrm{r} /$} \\
\cline { 2 - 5 } Rango etario & Sílabas aisladas & \multicolumn{3}{c}{ Palabras } \\
\cline { 2 - 5 } & $(100 \%=105)$ & $\begin{array}{c}\text { Ataque } \\
(100 \%=105)\end{array}$ & $\begin{array}{c}\text { Coda } \\
(100 \%=84)\end{array}$ & $\begin{array}{c}\text { Grupo consonántico } \\
(100=294)\end{array}$ \\
\hline $3.00-3.05$ & $56,2 \%$ & $57,1 \%$ & $27,7 \%$ & $27,4 \%$ \\
$3.06-3.11$ & $56,2 \%$ & $54,3 \%$ & $53,7 \%$ & $38,1 \%$ \\
$4.00-4.05$ & $83,8 \%$ & $91,4 \%$ & $81,8 \%$ & $71,4 \%$ \\
$4.06-4.11$ & $98,1 \%$ & $98,1 \%$ & $82,3 \%$ & $84,5 \%$ \\
\hline
\end{tabular}

En la Tabla 3, se observa que el fonema vibrante simple alcanza mayores porcentajes de producción correcta en sílaba aislada y en posición de ataque dentro de las palabras, aunque no es posible definir con claridad una secuencia de mayor a menor porcentaje entre los contextos, ya que estos cambian de orden según el rango etario. El fonema en posición de coda y en grupos consonánticos presenta un aumento sostenido de su porcentaje de emisión según avanza la edad de los sujetos, lo que también se observa en los otros dos contextos a partir de los
4 años. Al aplicar el criterio de adquisición del 80\%, se observa que los contextos de sílaba aislada y de palabra en posición de ataque y coda alcanzan el punto de corte a partir de los 4 años de edad, no así en grupos consonánticos, que lo alcanzan a partir de los 4 años 6 meses. Por el contrario, si se opta por el criterio del $90 \%$, los contextos de coda y grupo consonántico no evidenciarían adquisición antes de los 5 años de edad; en sílaba aislada estaría adquirido a partir de los 4.06 años; y en posición de ataque incluso antes, a partir de los 4 años. 
Tabla 4

Porcentajes de producción del fonema / r / por rango etario con distinción de contextos fonológicos (entre paréntesis se señala el número máximo de producciones en cada contexto)

\begin{tabular}{ccc}
\hline & & Fonema $/ \mathrm{r} /$ \\
\cline { 2 - 3 } Rango etario & Silabas aisladas & Palabra Ataque \\
\cline { 2 - 3 } & $(100 \%=210)$ & $(100 \%=42)$ \\
\hline $3.00-3.05$ & $17,1 \%$ & $14,3 \%$ \\
$3.06-3.11$ & $27,6 \%$ & $35,7 \%$ \\
$4.00-4.05$ & $66,7 \%$ & $78,6 \%$ \\
$4.06-4.11$ & $62,4 \%$ & $73,8 \%$ \\
\hline
\end{tabular}

La Tabla 4 evidencia que el fonema vibrante múltiple presenta mayor porcentaje de producción en palabras (ataque) que en sílabas aisladas, con excepción del rango etario de los 3.00 a los 3.05 años. En ambos contextos, además, se observan porcentajes crecientes de producción correcta hasta el grupo de 4 años y una posterior disminución. Finalmente, ambos contextos aparecen como no adquiridos antes de los 5 años de edad, ya sea que se ocupe el criterio del $80 \%$ o del $90 \%$.

\section{b) Resultados por sujetos}

A continuación se presentan los resultados del análisis de los datos centrado en los sujetos participantes. En primera instancia, la Tabla 5 ilustra el porcentaje de niños que producen los fonemas estudiados, en todos los contextos de aparición, según el criterio de evidencia de producción ("al menos lo dice una vez, incluido siempre").

Tabla 5

Cantidad de niños por rango etario que evidencian producción de los fonemas lateral y róticos, sin distinguir contexto fonológico ( $n=21$ por cada rango etario)

\begin{tabular}{cccc}
\hline Rango etario & I & $r$ & $r$ \\
\hline $3.00-3.05$ & $100 \%$ & $95,2 \%$ & $42,9 \%$ \\
$3.06-3.11$ & $100 \%$ & $90,5 \%$ & $61,9 \%$ \\
$4.00-4.05$ & $100 \%$ & $100 \%$ & $85,7 \%$ \\
$4.06-4.11$ & $100 \%$ & $100 \%$ & $85,7 \%$ \\
\hline
\end{tabular}

Tal como evidencia la Tabla 5 , los resultados muestran situaciones distintas para cada fonema. En primer lugar, todos los niños de la muestra producen el fonema / I /, ya sea una vez, varias veces o siempre. De igual manera ocurre con todos los niños de 4 años en relación con el fonema / r /. A los 3 años, hay más niños que elicitan este fonema en el rango de 3.00 a 3.05 años que en el rango de 3.06 a 3.11 años. En relación con / $r$ /, se observa un aumento en el número de niños que evidencia su producción desde los 3 años hasta los 4 años de edad, aunque hacia los 5 años aún hay niños que nunca producen correctamente el fonema. Al diferenciar los datos según el criterio del $80 \%$, se observa que tanto / I / como / r / estarían adquiridos desde los 3 años; en cambio / r / lo haría desde los 4 años. Si los datos se distinguen según el criterio del $90 \%$, no cambiaría el juicio en torno a los dos 
primeros fonemas, pero / $\mathrm{r} / \mathrm{no}$ alcanzaría el punto de corte antes de los 5 años.

En segunda instancia, la Tabla 6 muestra el porcentaje de niños que producen los fonemas estudiados, sin considerar el contexto fonológico, según la distinción entre inestabilidad y estabilidad del fonema ("al menos lo dice una vez, pero no siempre" versus "lo dice siempre", respectivamente).

Tabla 6

Cantidad de niños por rango etario que producen los fonemas lateral y róticos, sin distinguir contexto fonológico

\begin{tabular}{ccccccc}
\hline Rango etario & \multicolumn{2}{c}{ I } & \multicolumn{2}{c}{$r$} & \multicolumn{2}{c}{$r$} \\
\cline { 2 - 7 } & Inestabilidad & Estabilidad & Inestabilidad & Estabilidad & Inestabilidad & Estabilidad \\
\hline $3.00-3.05$ & $100 \%$ & $0,0 \%$ & $95,2 \%$ & $0,0 \%$ & $42,9 \%$ & $0,0 \%$ \\
$3.06-3.11$ & $90,5 \%$ & $9,5 \%$ & $85,7 \%$ & $4,8 \%$ & $57,1 \%$ & $4,8 \%$ \\
$4.00-4.05$ & $76,2 \%$ & $23,8 \%$ & $85,7 \%$ & $14,3 \%$ & $28,6 \%$ & $57,1 \%$ \\
$4.06-4.11$ & $76,2 \%$ & $23,8 \%$ & $71,4 \%$ & $28,6 \%$ & $38,1 \%$ & $47,6 \%$ \\
\hline
\end{tabular}

Este análisis de los datos permite varias observaciones interesantes. En primer lugar, ningún niño del rango de 3.00 a 3.05 años de edad logra decir siempre los tres fonemas del estudio en todos los contextos. En segundo lugar, al aumentar el rango etario se observa que para los fonemas lateral y vibrante simple disminuye el porcentaje de niños que los presentan inestables o en proceso de estabilización, a la vez que tiende a aumentar el de quienes los presentan estabilizados, situación que no se da con tanta claridad en el caso de la vibrante múltiple. Por último, destaca el hecho de que en los rangos etarios superiores haya más niños que presentan estabilizados los fonemas vibrantes a diferencia de la lateral.

Como se indicó anteriormente, algunos estudios trabajan con el criterio de que un niño tiene el fonema cuando lo dice siempre que se le solicita, es decir, con un criterio de estabilización similar al utilizado en esta investigación. Si sobre los porcentajes de niños que cumplen con esa condición, se aplica el criterio grupal del $80 \%$ y del $90 \%$, los datos arrojarían que ninguno de los fonemas en estudio alcanza niveles de adquisición en ningún rango etario. En otras palabras, en ningún tramo de edad más del $80 \%$ o del $90 \%$ de los niños logra manifestar de manera estabilizada los fonemas / I /, / r / y / r /.

\section{c) Comparación de análisis por fonema y análisis} por niño

A continuación, la Tabla 7 ilustra una comparación entre los resultados obtenidos al realizar los análisis centrados tanto en el fonema como en el niño. 
Tabla 7

Edad en la que cada fonema alcanzaría la categoría de adquirido según los distintos criterios utilizados

\begin{tabular}{|c|c|c|c|c|c|c|c|c|}
\hline \multirow{2}{*}{$\begin{array}{l}\text { Unidad de } \\
\text { análisis }\end{array}$} & \multirow{2}{*}{\multicolumn{2}{|c|}{ Criterio de análisis }} & \multicolumn{2}{|c|}{1} & \multicolumn{2}{|c|}{$r$} & \multicolumn{2}{|c|}{$r$} \\
\hline & & & $80 \%$ & $90 \%$ & $80 \%$ & $90 \%$ & $80 \%$ & $90 \%$ \\
\hline \multirow{5}{*}{$\begin{array}{c}\text { Por } \\
\text { fonema }\end{array}$} & $\%$ de & En todos los contextos & 4.00 & 4.00 & 4.06 & $*$ & $*$ & $*$ \\
\hline & emisiones & En sílaba aislada & 3.00 & 4.00 & 4.00 & 4.06 & * & $*$ \\
\hline & correctas del & En posición de ataque & 4.00 & 4.00 & 4.00 & 4.00 & $*$ & $*$ \\
\hline & fonema por & En posición de coda & 4.00 & $*$ & 4.00 & $*$ & & \\
\hline & rango etario & En grupo consonántico & 4.00 & 4.00 & 4.06 & $*$ & & \\
\hline \multirow{2}{*}{ Por niño } & $\begin{array}{l}\% \text { de niños que } \\
\text { producen el }\end{array}$ & $\begin{array}{l}\text { Al menos una vez } \\
\text { (evidencia de producción) }\end{array}$ & 3.00 & 3.00 & 3.00 & 3.00 & 4.00 & $*$ \\
\hline & $\begin{array}{l}\text { fonema por } \\
\text { rango etario }\end{array}$ & Siempre (estabilización) & $*$ & $*$ & $*$ & $*$ & $*$ & $*$ \\
\hline
\end{tabular}

* Bajo dicho criterio de análisis, no logra establecerse un rango etario entre los 3.00 y los 4.11 años de edad como adquirido.

Los datos muestran que la etiqueta de adquirido puede cambiar de manera importante según el conjunto de criterios utilizados. En el caso de / I /, en la mayoría de los contextos de producción alcanza el punto de adquirido a los 4 años, excepto en posición de coda silábica cuando el criterio es el del 90\%; por el contrario, en el análisis centrado en los sujetos, la edad de adquisición varía de los 3 años para la evidencia de producción hasta más allá de los 5 años (es decir, fuera de los rangos de edad del estudio) para el criterio de estabilidad. Lo mismo pasa con la vibrante simple cuando el procesamiento de los datos se centra en los sujetos; en cambio, cuando el procesamiento está centrado en el fonema, aparecen más contextos en que el punto de adquisición no se alcanza en las edades estudiadas bajo un criterio del $90 \%$. Por último, el caso de la vibrante múltiple es quizás el menos cambiante, pues bajo ningún criterio, en ningún contexto, alcanza el punto de adquisición, excepto si se considera el criterio de evidencia de producción sobre el $80 \%$ de los niños, en que aparece adquirido en el rango que va de los 4.00 a los 4.05 años de edad.

\section{Discusión}

Tal como muestran los resultados de la presente investigación, existen diferencias importantes en la determinación de la edad de adquisición, según los distintos criterios con los que se analizan los datos. Se observan discrepancias que pueden estimarse incluso mayores de dos años para un mismo fonema, específicamente en el caso de / r /, al comparar un análisis centrado en el fonema con un análisis centrado en los sujetos. Debido a estas diferencias, resulta fundamental explicitar, a la hora de estudiar edades de adquisición, todo el conjunto de criterios utilizados, desde las definiciones teóricas del fenómeno hasta las decisiones prácticas a la hora de procesar los datos.

En este trabajo, se han identificado tres aspectos muy relevantes de explicitar cuando se investiga acerca del desarrollo fonético-fonológico. El primero tiene relación con lo que se ha definido como el foco en el procesamiento de los datos: el fonema o el niño. Las investigaciones centradas en el fonema (Acevedo, 1993; Melgar, 1976/2007; Serra, 1984; Vivar, 2009; Vivar y León, 2009), explícita o implícitamente, conciben que cada sonido presenta 
características inherentes a él que propician su adquisición más temprana o más tardía en la población infantil, los que son descritos en términos de rasgos, tales como modo o punto. En estos casos, se asume que los resultados presentan cierta autonomía en relación con los factores sociodemográficos de los participantes. Por el contrario, si el procesamiento de los datos se centra en los participantes, se asume que cada uno de ellos podría presentar características que facilitan o no el dominio de un sonido (Bosch, 1983), tales como condiciones de desarrollo, habilidades de discriminación auditiva o prácticas de interacción comunicativa. Sin embargo, ninguna de las investigaciones referida al español que trabaja con esta perspectiva analiza sus resultados a la luz de estas variables individuales.

El segundo aspecto tiene que ver con la concepción que se tiene del fenómeno de adquisición y cómo se operacionaliza: ¿Es un fenómeno continuo y progresivo?, ¿cómo se establece que un elemento lingüístico ya está adquirido? Existe una suerte de disyuntiva no resuelta en relación con el estatus de adquirido de un elemento, en cuanto a si se entiende como la evidencia de uso de un fonema, aunque el niño presente una producción muy inestable del mismo, o bien, como la estabilización completa en todos los contextos y usos. En este sentido, puede resultar útil la distinción que realizan algunos trabajos entre emergencia, uso habitual, adquisición y dominio de un fonema, aunque cabe destacar que tampoco existe una completa unificación de criterios para establecer dichas categorías entre los distintos autores: Másdóttir y Stokes (2015) distinguen entre emergence, acquisition y mastery según el porcentaje de emisiones correctas de cada fonema del islandés por cada grupo de edad (sobre el 50\%, sobre el $75 \%$ y sobre el $90 \%$, respectivamente). EI mismo criterio ocupan para el árabe Amayreh y Dyson (1998), aunque a la primera categoría la denominan age of customary production. MacLeod, Sutton, Trudeau y Thordardottir (2010), por su parte, distinguen, para el francés de Quebec, entre customary, acquisition y mastery según el porcentaje de niños que presenta un fonema (sobre 50\%, 75\% y $90 \%$, respectivamente), al menos en dos posiciones dentro de la palabra, para customary, o en todas las posiciones posibles, para acquisition y mastery. De igual manera proceden San Phoon, Abdullah, Wah Lee y Murugaiah (2014), para el malayo, aunque los porcentajes citados deben observarse en dos grupos de edades consecutivos para definir la condición de un fonema. Por último, MacLeod et al. (2011) además distinguen entre emergency y accurately, según si el fonema es emitido, al menos una vez, por menos o más del $75 \%$ de los niños de la muestra respectivamente, con independencia de que el fonema estuviera o no presente en la palabra adulta que el niño intentaba producir. Lo mismo hace Zhu (2002), en una investigación de chino mandarín, aunque cuando el fonema es producido por más del $75 \%$ de los niños lo denomina phoneme stabilization. Más allá de las diferencias entre los autores, estos trabajos ofrecen posibilidades de analizar los datos de maneras más complejas y variadas que la simple dicotomización entre adquirido/no adquirido, lo que permitiría dar cuenta de que el proceso descrito es progresivo, incluso en investigaciones transversales. 
En el caso de las investigaciones centradas en el fonema, el estatus de adquirido debe tomarse, además, con cuidado, pues dichos estudios representan resultados grupales y no individuales de producción. En ese sentido, no sería correcto interpretar que todos los individuos de un grupo se comportan como la tendencia del grupo y, por lo tanto, es un error proyectar los resultados de investigaciones con este enfoque en la descripción de niños particulares, sobre todo si es para tomar una decisión terapéutica. Este tipo de razonamiento indebido es conocido como falacia ecológica (Bonita, Beaglehole y Kjellström, 2008).

El aspecto operacional de este punto tiene que ver con la utilización de un porcentaje de referencia que indica que un elemento se encuentra o no adquirido. Las investigaciones citadas anteriormente coinciden en utilizar el $90 \%$ de producciones grupales o de sujetos por grupo para indicar que un fonema alcanzó el nivel de dominio. Para Dodd et al. (2003), la elección de un porcentaje específico se basa en cómo fue seleccionada la muestra. En su caso, no hubo criterios de exclusión de participantes según criterios lingüísticos, sino que se tomó un diseño muestral aleatorio para incluir a participantes de diversas características. Lo anterior justifica para ellas que, al momento de ofrecer edades de referencia, se utilice el criterio del $90 \%$, pues se asume que hasta un $10 \%$ de la población puede presentar algún tipo de dificultad articulatoria o desorden de habla. No todas las investigaciones en español, sin embargo, utilizan dicho punto de corte y en el caso de las que lo usan, no explicitan una justificación teórica para optar por un 65\%, 75\%, $80 \%, 90 \%$ o 100\%, aunque todas ellas han seleccionado previamente a los niños de la muestra, con el fin de que los datos representen a la población sin dificultades de lenguaje y/o habla y presenten ciertas características demográficas (Vivar y León, 2007). Para esto, en varios casos se utilizó una evaluación fonoaudiológica, no siempre explícita en cuanto a sus características, lo que conlleva además, la paradoja implícita de que los niños pueden haber sido seleccionados o descartados con criterios que justamente se buscaba conocer a través de estas investigaciones. Sumado a lo anterior, ¿qué justifica el uso de un porcentaje cualquiera, cuando la muestra con la que se trabaja ya presenta criterios de exclusión y se intenta dar cuenta de un fenómeno típico? Este no es un problema exclusivo de las investigaciones en español, ya que Ferrante, Van Borsel y Madeiros de Britto (2008), en un estudio para el portugués brasileño, utilizaron el umbral del $75 \%$, también sin mayor justificación. Resulta evidente que en este punto hace falta una discusión profunda acerca de los alcances, las limitantes y las implicancias teóricas, prácticas y éticas de trabajar con los conceptos de desarrollo típico o de normalidad estadística (Silva, 1997), de adquisición como proceso y de estabilidad, inestabilidad y emergencia en las etapas de desarrollo.

El último aspecto se refiere a los contextos fonológicos en que aparece el fonema en estudio. Los resultados muestran que la edad en que se considere adquirido un elemento puede variar de manera importante si se utilizan todos los contextos silábicos a la vez, o se analizan los contextos separados por separado (ver Tabla 7). Lo anterior implica que se debe observar con mucha atención qué contextos son incluidos al ofrecer una edad de 
Torres, F., Pino, J., Peñaloza, C., Rojas, D., Fernández, MA., Castro, P., Bustos, M. y Hormazábal, K.

referencia y cuáles se están considerando al momento de, por ejemplo, evaluar a un niño en específico. Una referencia que repare en todos los contextos, incluido el fonema en posición de coda y/o conformando grupo consonántico, puede resultar más severa que uno que surja solo del análisis del fonema en posición de ataque.

En resumen, las distintas investigaciones ofrecen diferentes criterios para procesar el dato fonéticofonológico obtenido a partir de sus muestras y, por lo tanto, hacen referencia a fenómenos distintos cuando entregan sus resultados. Lo anterior hace evidente que las conclusiones de estas investigaciones no son necesariamente comparables entre sí, aunque hubiesen escogido instrumentos y métodos de recolección de las muestras idénticos. Además, no siempre se ofrece la discusión teórica para justificar la preferencia de un método de análisis por sobre otro y evidenciar las implicancias de dichas opciones; es decir, los criterios utilizados resultan arbitrarios y podría optarse por uno o por otro, indistintamente. Esto conlleva el problema de proyectar información que no es posible aseverar a partir de los datos, como ha venido sucediendo en la práctica fonoaudiológica en Chile, donde se utilizan estudios normativos de adquisición de fonemas y se consideran estas edades como referencia para tomar decisiones terapéuticas. Desde una perspectiva teórica, se evidencia que aún está pendiente en español una reflexión profunda y explícita respecto de la naturaleza de los procesos de adquisición y desarrollo fonético-fonológico, y acerca de la influencia e interacción de la conducta observada con otros aspectos lingüísticos y del entorno de los niños, como sus habilidades auditivas o su entorno socioambiental, los que suelen trabajarse como criterios de inclusión o exclusión, pero sin una discusión de los resultados a la luz de dichos rasgos.

\section{Referencias}

$\begin{array}{lrrr}\text { Acevedo, M. A. (1993). Development of } & \text { Spanish } \\ \text { consonants in preschool children. } & \text { Childhood } \\ \text { Communication } & \text { Disorders, } & 15(2), & 9-15 . \\ \text { http://dx.doi.org/10.1177/152574019301500202 } & \end{array}$

Amayreh, M. \& Dyson, A. (1998). The acquisition of Arabic consonants. Journal of Speech, Language, and Hearing Research, 41(3), 642-653. http://dx.doi.org/10.1044/jslhr.4103.642

Bermeosolo, J. (2001). Psicología del lenguaje. Santiago de Chile: Ediciones Universidad Católica de Chile.

Bonita, R., Beaglehole, R., y Kjellström, T. (2008). Epidemiología básica. Washington D.C.: Organización Panamericana de la Salud.

Bosch, L. (1983). El desarrollo fonológico infantil: una prueba para su evaluación. Anuario de Psicología, 28(1), 86-114.

Camargo, M. (2006). Desarrollo fonético-fonológico del español en niños. Umbral Científico, 9, 5-9.

Dodd, B., Holm, A., Hua, Z., \& Crosbie, S. (2003). Phonological development: A normative study of British English-speaking children. Clinical Linguistics \& Phonetics, 17(8), 617-643. http://dx.doi.org/10.1080/0269920031000111348

Ferrante, C., Van Borsel, J., \& Madeiros de Britto Pereira, M. (2008). Aquisiçao fonológica de crianças de clase sócio economica alta. Revista CEFAC, 10(4), 452-460. http://dx.doi.org/10.1590/s1516-18462008000400005

Hormazábal, K., Larrea, R., Muñoz, M., Ruz, M., y Toro, P. (2013). Edad de adquisición de fonemas líquidos en un grupo de niños chilenos de entre 3 y 4 años 11 meses de nivel socioeconómico medio en la Región Metropolitana (Seminario de Licenciatura en Fonoaudiología). Universidad de Chile, Santiago, Chile. 
Jara, A., Poblete, D., Riquelme, F., Salgado, L., y Zúñiga, C. (2014). Edad de adquisición de fonemas líquidos en un grupo de niños de chilenos entre 3 y 5.11 años de nivel socioeconómico bajo en la Región Metropolitana (Seminario de Licenciatura en Fonoaudiología). Universidad de Chile, Santiago, Chile.

MacLeod, A., Sutton, A., Trudeau, N., \& Thordardottir, E. (2010). The acquisition of consonants in Québécois French: A cross-sectional study of pre-school aged children. International Journal of Speech-Language Pathology, 13(2), 93-109. http://dx.doi.org/10.3109/17549507.2011.487543

Másdóttir, T. \& Stokes, S. (2015). Influence of consonant frequency on Icelandic-speaking children's speech acquisition. International Journal of Speech-Language Pathology, 18(2), 111-121. http://dx.doi.org/10.3109/17549507.2015.1060525

Melgar, M. (1976/2007). Cómo detectar al niño con problemas del habla ( $5^{\circ}$ ed.). Ciudad de México: Editorial Trillas.

San Phoon, H., Abdullah, C., Wah Lee, L., \& Murugaiah, P. (2014). Consonant acquisition in the Malay language: A cross-sectional study of preschool aged Malay children. Clinical Linguistics \& Phonetics, 28(5), 329-345. http://dx.doi.org/10.3109/02699206.2013.868517

Serra, M. (1984). Normas estadísticas de articulación para la población escolar de 3 a 7 años del área metropolitana de Barcelona. Revista de Logopedia, Foniatría y Audiología, $3(4), \quad 232-235 . \quad$ http://dx.doi.org/10.1016/s02144603(83)75286-1

Silva, L. C. (1997). Cultura estadística e investigación científica en el campo de la salud: una mirada crítica. Madrid: Ediciones Díaz de Santos.

Susanibar, F., Dioses, A., y Huamaní, O. (2013). Fundamentos para la evaluación de las alteraciones del habla de origen fonético-fonológico. En F. Susanibar, D. Parra, y A. Dioses (Coords.), Motricidad Orofacial: fundamentos basados en evidencias. Madrid: EOS.

Vivar, P. (2009). Evaluación de grupos consonánticos de ataque complejo en un grupo de niños de la ciudad de Concepción con prueba articulatoria CEFI. Onomázein, 20(2), 33-44. Recuperado de http://www.redalyc.org/html/1345/134512612002/
Vivar, P. y León, H. (2007). Aplicación del Cuestionario para la Evaluación de la Fonología Infantil (C.E.F.I.) a una muestra de niños chilenos. Revista Chilena de Fonoaudiología, 8, 17-31. http://dx.doi.org/10.5354/0719$\underline{4692.2007 .21378}$

Vivar, P. y León, H. (2009). Desarrollo fonológico-fonético en un grupo de niños entre 3 y 5,11 años. Revista CEFAC, 11(2), 190-198. http://dx.doi.org/10.1590/s1516$\underline{18462009000200003}$

Zhu, H. (2002). Phonological development in specific contexts: Studies of Chinese-speaking children. Clevedon: Multilingual Matters. 